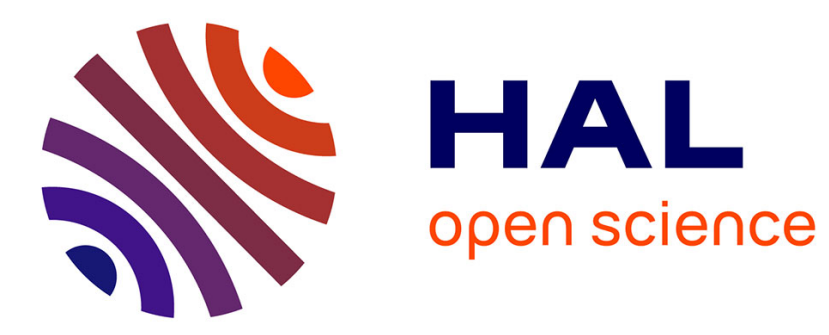

\title{
Construction d'un spectrofluorimètre corrigé en analyse et en excitation
}

\author{
G. Normandin, P. Donnat, R. Journeaux
}

\section{To cite this version:}

G. Normandin, P. Donnat, R. Journeaux. Construction d'un spectrofluorimètre corrigé en analyse et en excitation. Revue de Physique Appliquée, 1971, 6 (2), pp.147-153. 10.1051/rphysap:0197100602014700 . jpa-00243515

\section{HAL Id: jpa-00243515 https://hal.science/jpa-00243515}

Submitted on 1 Jan 1971

HAL is a multi-disciplinary open access archive for the deposit and dissemination of scientific research documents, whether they are published or not. The documents may come from teaching and research institutions in France or abroad, or from public or private research centers.
L'archive ouverte pluridisciplinaire HAL, est destinée au dépôt et à la diffusion de documents scientifiques de niveau recherche, publiés ou non, émanant des établissements d'enseignement et de recherche français ou étrangers, des laboratoires publics ou privés. 


\title{
CONSTRUCTION D'UN SPECTROFLUORIMẼTRE CORRIGÉ EN ANALYSE ET EN EXCITATION
}

\author{
par G. NORMANDIN, P. DONNAT et R. JOURNEAUX \\ Laboratoire de Physico-Chimie des Pigments Végétaux et des Substances Modèles \\ Ecole Normale Supérieure de Saint-Cloud, 92, Saint-Cloud
}

(Reçu le 7 janvier 1971)

\begin{abstract}
Résumé. - Nous avons construit un spectrofluorimètre réalisant une correction automatique au niveau de l'excitation et de l'analyse.

L'énergie de la lumière d'excitation est maintenue constante au niveau de la cellule de fluorescence par l'utilisation d'une thermopile. Les variations de la fonction d'appareil du système d'analyse sont compensées au niveau de l'enregistrement du signal de fluorescence.
\end{abstract}

Abstract. - A spectrofluorimeter has been achieved which performs automatic corrections relatively to excitation and analysis. The excitation light falling on the fluorescence cell has an energy kept constant with a thermopile. The variations of the sensibility of analysis apparatus are compensated when recording fluorescence signal.

En Chimie et en Biologie, l'étude de la fluorescence présente un intérêt comparable aux techniques d'absorption. Mais alors que ces dernières ont conduit à l'élaboration d'appareils commerciaux permettant des mesures absolues, la plupart des fluorimètres ne possèdent aucun système de correction et ne sont utilisables que pour des expériences comparatives. Nous avons construit un appareil permettant d'effectuer automatiquement les corrections dues aux fonctions d'appareil et aux fluctuations dans le temps de la source lumineuse. D'autre part, nous avons choisi certaines composantes de façon à obtenir une sensibilité suffisante en analyse entre 6000 et $8000 \AA$, région de fluorescence des chlorophylles et de nombreux appareils photosynthétiques.

I. Etude théorique. - 1. CoRreCtions NÉCESSAIRES EN FLUORESCENCE. - La réponse d'un fluorimètre $\rho\left(v_{F}\right)$ est le produit de deux termes $\rho_{1}$ et $\rho_{2}$, le premier caractérisant l'excitation, le deuxième la détection de la lumière de fluorescence [1].

Ces deux termes ont comme valeur :

$$
\begin{aligned}
& \rho_{1}=\Phi\left(v_{E}\right) \cdot \mathrm{e}^{-\varepsilon_{E} c a}\left(1-\mathrm{e}^{-\varepsilon_{E} c b}\right) \\
& \rho_{2}=h \cdot Q\left(v_{F}\right) \cdot v_{F} \cdot T\left(v_{F}\right) \cdot R
\end{aligned}
$$

$\Phi\left(v_{E}\right)=$ flux lumineux photonique arrivant sur la cuve à fluorescence de fréquence $v_{E}$ (longueur d'onde $\lambda_{E}$ ).

$\varepsilon_{E}=$ coefficient d'absorption molaire de la solution pour la longueur d'onde d'excitation $\lambda_{E}$.

$c=$ concentration de la substance fluorescente (on considère que la seule substance qui fluoresce est celle qui absorbe). $a$ et $b$ sont les côtés délimitant les parties du faisceau (Fig. 1).

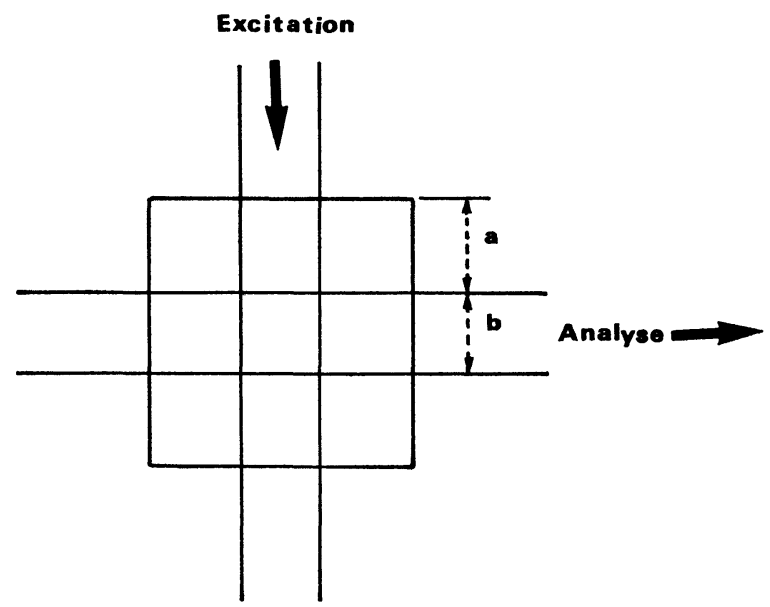

FIG. 1. - Géométrie des faisceaux lumineux dans la cuve à fluorescence.

$h=$ facteur géométrique tenant compte du fait qu'une partie seulement de la lumière émise est analysée. Pour un appareil donné, si on néglige les aberrations chromatiques, $h$ ne dépend que de l'ouverture des fentes du monochromateur d'analyse.

$Q\left(v_{F}\right)=$ rendement quantique de fluorescence pour la fréquence d'analyse $v_{F}$ (longueur d'onde $\lambda_{F}$ ) et pour la fréquence d'excitation $v_{E}$.

$T\left(v_{F}\right)=$ facteur de transmission énergétique de l'ensemble d'analyse.

C'est le courant anodique du photomultiplicateur d'analyse pour un flux analysé unité. 
$R=$ résistance de charge du photomultiplicateur. Dans cette expression on a négligé tout phénomène de réabsorption de la lumière émise.

On constate que la fonction $Q\left(v_{F}\right)$ qui caractérise la substance en solution n'est pas proportionnelle, quelles que soient les conditions, à la fonction expérimentale $\rho\left(v_{F}\right)$. Deux corrections sont donc à prévoir correspondant à chaque terme.

2. Correction en excitation. - Pour une solution donnée, la correction due au terme $\mathrm{e}^{-\varepsilon_{E} c a}\left(1-\mathrm{e}^{-\varepsilon_{E} c b}\right)$ peut se faire par le calcul dans le cas d'un spectre d'action. Nous ne discuterons donc que le rôle de $\Phi\left(v_{E}\right)$. Cette quantité varie dans le temps et en fonction de $v_{E}$ par suite respectivement des fluctuations de la source, de sa répartition spectrale et du pouvoir de transmission du monochromateur d'excitation. On peut maintenir constant le flux énergétique $\Phi\left(v_{E}\right) \cdot v_{E}$ par un système d'asservissement soit en agissant sur la source [2], [3] soit sur les fentes du monochromateur [4]. Une autre solution consiste à déterminer $\Phi\left(v_{E}\right)$ par un compteur quantique et à faire le quotient de deux tensions [5], [6], [7]. Nous avons retenu le système d'asservissement, la variation de $\Phi\left(v_{E}\right) . v_{E}$ étant compensée par la variation d'absorption d'un dégradé angulaire placé à la sortie du monochromateur d'excitation.

3. Correction en ANAlySE. - Le facteur de transmission $T\left(v_{F}\right)$ est une fonction de la longueur d'onde d'analyse $\lambda_{F}$. Il est le produit de la transmission du monochromateur d'analyse $\tau\left(v_{F}\right)$ et du gain du photomultiplicateur $I\left(v_{F}\right), I\left(v_{F}\right)$ étant le courant anodique pour un flux énergétique unité arrivant sur la photocathode. Si $\Phi_{F}\left(v_{F}\right)$ est le flux énergétique de la lumière de fluorescence entrant dans le monochromateur d'analyse, l'intensité du courant anodique vaut

$$
\Phi_{F}\left(v_{F}\right) \cdot \tau\left(v_{F}\right) \cdot I\left(v_{F}\right) \text {. }
$$

En général, on enregistre la chute de tension aux bornes d'une résistance de charge $R$ donc

$$
\rho\left(v_{F}\right)=R \cdot \Phi_{F}\left(v_{F}\right) \cdot \tau\left(v_{F}\right) \cdot I\left(v_{F}\right) .
$$

Seul le produit $\tau\left(v_{F}\right) \cdot I\left(v_{F}\right)=T\left(v_{F}\right)$ est en général déterminé expérimentalement et fait l'objet de la correction soit par le calcul, soit par un moyen électronique automatique [5], [6], [8].

La méthode que nous avons choisie consiste à compenser les variations de $T\left(v_{F}\right)$ par une résistance de charge $R$ variable.

II. Réalisation expérimentale. - 1. ScHÉMA DU FLUORIMÈTRE (Fig. 2). - La lumière excitatrice est fournie par un ensemble comprenant :

- Une source lumineuse (lampe quartz-iode Sylvania FAD $650 \mathrm{~W}$, température de couleur 3400 K, ou lampe à Xénon XBF OSRAM).

Les deux lampes sont alimentées en continu.

- Un monochromateur à réseau du type Hebert Fastié, focale $480 \mathrm{~mm}$, équipé d'un réseau Bausch et
Lomb de $52 \times 52 \mathrm{~mm}$, comportant 600 traits par $\mathrm{mm}$ et blazé à $5000 \AA$.

La lumière de fluorescence est analysée par un ensemble comprenant :

- Un monochromateur type Barr et Stroud, en montage Czerny Turner, dont la première moitié seulement a été utilisée. Il est équipé d'un réseau Jobin Yvon de $56 \times 56 \mathrm{~mm}$, comportant 1200 traits par mm et blazé à $5000 \AA$.

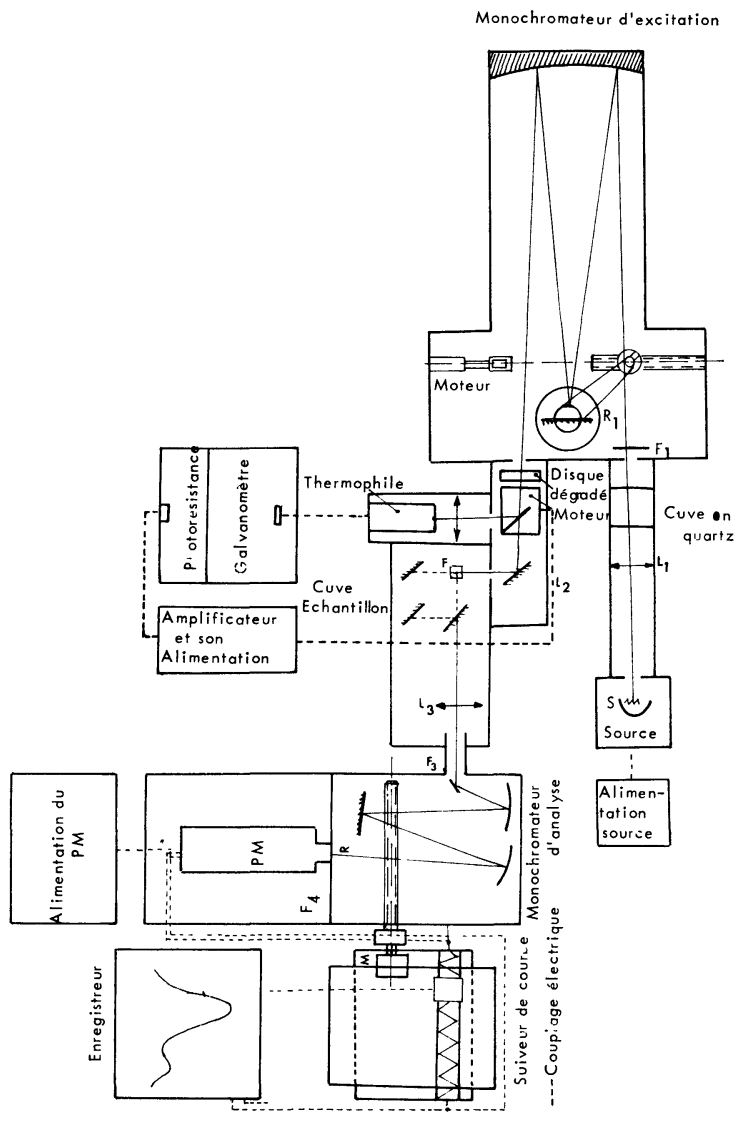

FIG. 2. - Schéma général du spectrofluorimètre.

- Un photomultiplicateur EMI 9558 B ayant une réponse spectrale du type $\mathrm{S} 20$. Le courant du photomultiplicateur est enregistré par l'intermédiaire de la tension apparaissant aux bornes d'une résistance de charge.

Les longueurs d'onde d'analyse et d'excitation d'une part, le papier de l'enregistreur d'autre part, sont entraînés linéairement en fonction du temps par des moteurs synchrones; les enregistrements sont donc directement gradués en longueur d'onde.

Le compartiment cuve a été étudié de façon à permettre une grande variété d'utilisation (Fig. 3). Son centre est conjugué à la fois à la fente de sortie du monochromateur d'excitation et de la fente d'entrée du monochromateur d'analyse par deux condenseurs en quartz. Un jeu de miroirs permet l'observation classique à $90^{\circ}$, par transmission, par réflexion oblique ou normale avec des cuves à fluorescence carrées $(10 \times 10 \mathrm{~mm})$. Le dernier mode a été aussi utilisé pour 
des solutions très absorbantes avec une cuve à épaisseur variable (cuve Mimosi de Jobin Yvon). Enfin des enregistrements à basse température sont possibles grâce à un vase spécial (accessoire Bayonne de Jobin Yvon).

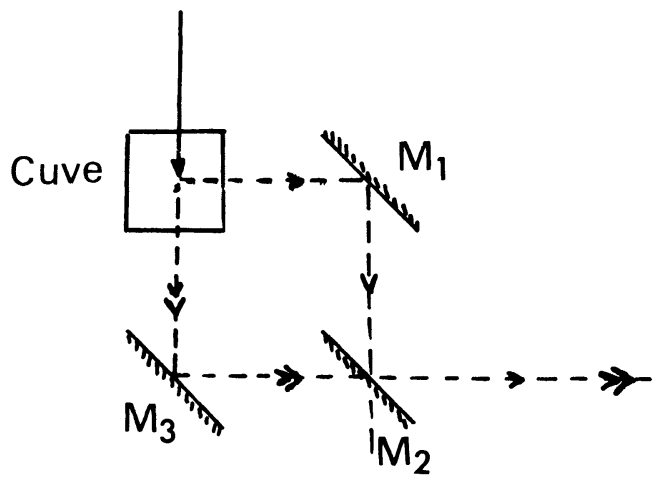

(a)

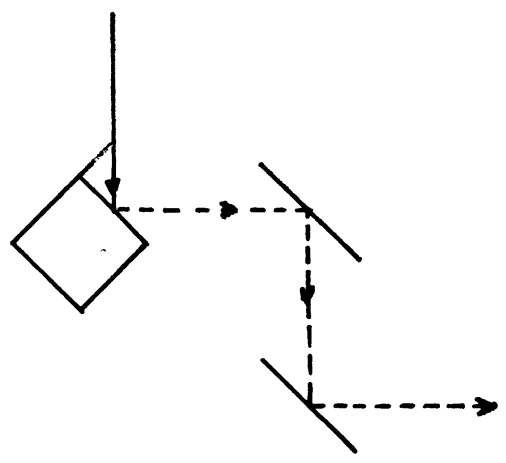

(b)

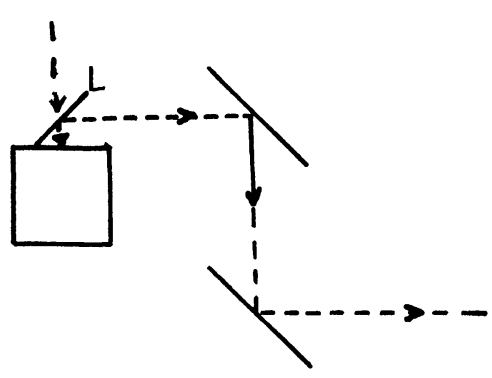

(c)

FIG. 3. - Utilisations du compartivement cuve : $a$ ) observation à $90^{\circ}$ (avec miroir $\mathrm{M}_{1}$ et $\mathrm{M}_{2}$ ) ou par transmission (avec miroir $\left.\mathrm{M}_{3}\right)$; b) observation par réflexion oblique ; $c$ ) observation par réflexion normale $(\mathrm{L}=$ lame semi-transparente).

2. Correction D'excitation. - Elle consiste à contrôler le flux énergétique arrivant sur la cuve à fluorescence par un système d'asservissement (Fig. 4).

L'organe de contrôle est constitué par un disque dégradé angulaire placé à la sortie du monochromateur d'excitation, il peut être entraîné par un moteur à double sens de rotation; on obtient ainsi une absorption variable.

L'organe de commande est constitué par une thermopile recevant une fraction constante du flux arrivant sur la cuve à fluorescence. Cette division est réalisée grâce à trois lames semi-transparentes identiques ; les deux faisceaux utilisés ont donc des énergies égales quelles que soient les variations des propriétés des lames.

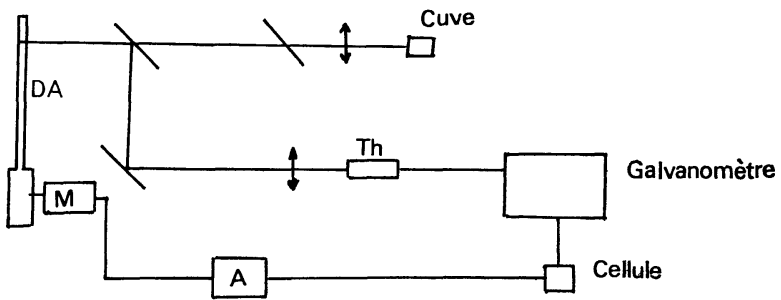

FIG. 4. - Contrôle du flux d'excitation : A : amplificateur d'asservissement; $M$ : moteur d'entraînement du dégradé angulaire DA; th : thermopile.

Le courant de la thermopile est détecté grâce à un galvanomètre dont le spot est focalisé sur une cellule photorésistante différentielle utilisée dans certains suiveurs de spots (Fig. 5). Elle est constituée par deux résistances $R p_{1}$ et $R p_{2}$ variables avec l'éclairement

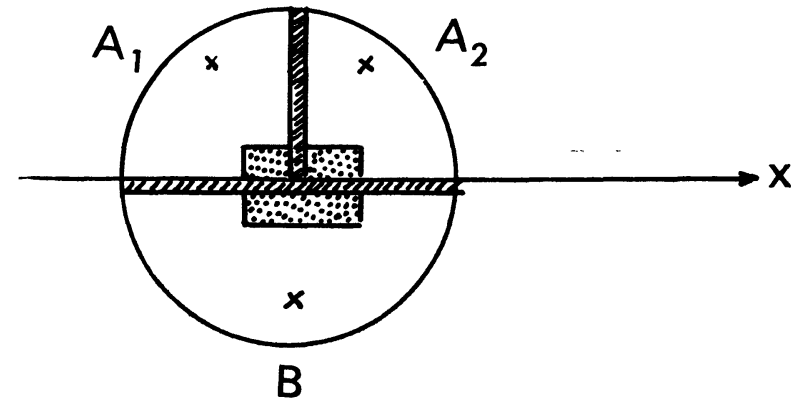

FIG. 5. - Cellule photorésistante différentielle : $R p_{1}:$ résistance entre $\mathrm{A}_{1}$ et $\mathrm{B} ; \boldsymbol{R} p_{2}$ : résistance entre $\mathrm{A}_{2}$ et $\mathrm{B}$.

qu'elles reçoivent. Ces deux résistances sont placées dans un pont de Wheatstone dont la tension de déséquilibre commande, par l'intermédiaire d'un amplificateur d'asservissement classique, le moteur couplé au disque dégradé (Fig. 6). Le sens de rotation du moteur dépend du sens du déséquilibre du pont, donc de la variation du rapport $R p_{1} / R p_{2}$, donc de la place du spot sur la cellule.

Le pont est équilibré, donc le disque est immobile, quand $R p_{1}=R p_{2}$, c'est-à-dire quand le spot du galvanomètre éclaire symétriquement la cellule photorésistante ; c'est la position de repos. La cellule photorésistante étant fixe, toute variation du flux lumineux sortant du monochromateur d'excitation va provoquer un déplacement du spot du galvanomètre, donc va faire varier le rapport $R p_{1} / R p_{2}$; cela va entraîner la rotation du disque dégradé dans un sens tel que la variation d'absorption compense la variation initiale du flux. Le réducteur de vitesse adapté au moteur a été choisi de façon à obtenir une réponse suffisamment rapide, tout en évitant les oscillations. Il faut également éviter les variations trop rapides de flux ; en effet, si le spot quitte la cellule photorésistante, la stabilisation n'existe plus. 

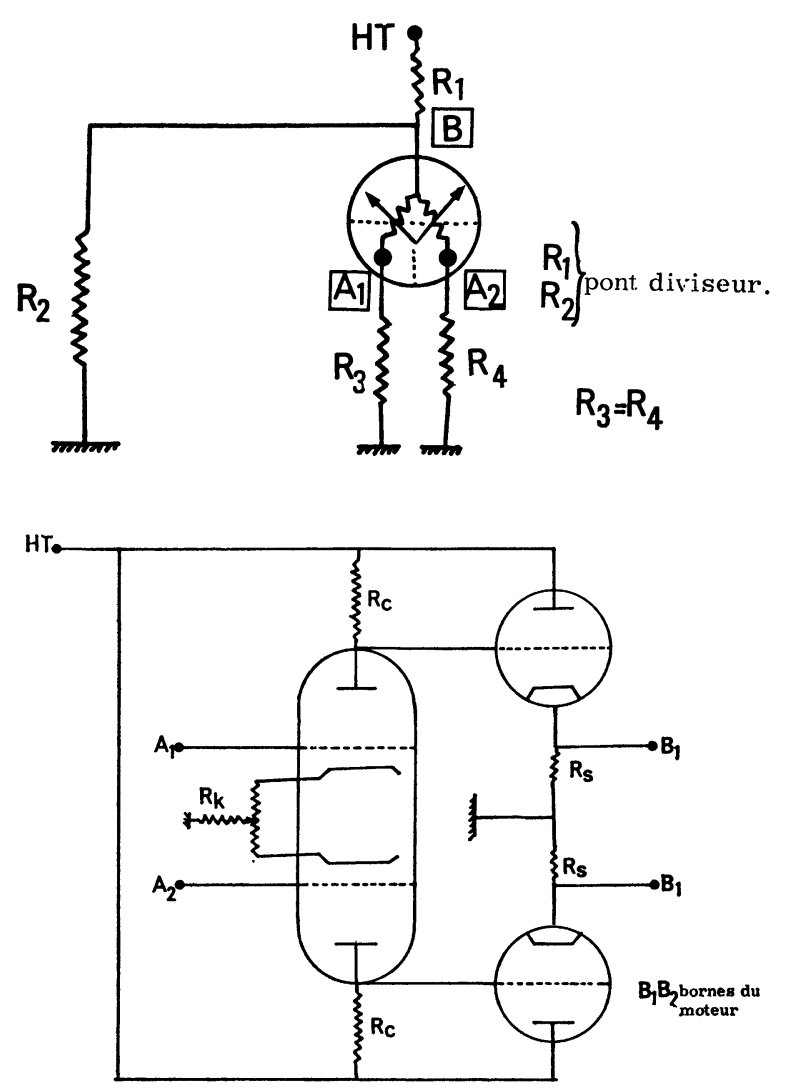

FIG. 6. - Amplificateur d'asservissement.

3. Correction d'analyse. - Elle doit tenir compte du pouvoir de transmission $T\left(v_{F}\right)$ de l'ensemble d'analyse de la lumière de fluorescence.

Détermination de la fonction $T\left(v_{F}\right)$. - Nous utilisons comme source la lumière arrivant sur le compartiment cuve et possédant une énergie constante quelle que soit la longueur d'onde. Toute cette lumière est envoyée par un miroir dans l'ensemble d'analyse et nous enregistrons, par balayage de la longueur d'onde $\lambda_{F}$, une courbe dont l'aire est proportionnelle à $T\left(v_{F}\right)$.

Principe de la correction. - Soit $R$ la résistance de charge du P. M. La réponse de l'ensemble d'analyse sera $: \rho\left(v_{F}\right)=R . T\left(v_{F}\right) . \Phi_{F}\left(v_{F}\right)$. Pour que

$$
\rho\left(v_{F}\right)=K . \Phi_{F}\left(v_{F}\right)
$$

$K$ étant une constante, il faut prendre pour $R$ une fonction de $v_{F}$ (ou de $\lambda_{F}$ ) telle que $R\left(v_{F}\right) \cdot T\left(v_{F}\right)=K$.

Réalisation pratique (Fig. 7). - Il faut réaliser une résistance variable de la forme $R\left(v_{F}\right)=\frac{K}{T\left(v_{F}\right)}$; ceci pourrait être conçu avec une série de potentiomètres, mais nous avons préféré une solution donnant à $R\left(v_{F}\right)$ une variation continue. La résistance variable peut être considérée comme un rhéostat dont le point de contact variable $C$ serait lié à un dispositif suiveur de courbes; on a alors $R=B C$. Si $B C=\frac{K}{T\left(v_{F}\right)} R$ aura bien une valeur correcte.
La courbe $B C=\frac{K}{T\left(v_{F}\right)}$ est tracée sur un rouleau de papier entrâné par le même moteur que le balayage en longueur d'onde du monochromateur d'analyse, le point $C$ étant solidaire de la cellule du suiveur de courbe. Pour tracer cette courbe on donne à $B C$ la

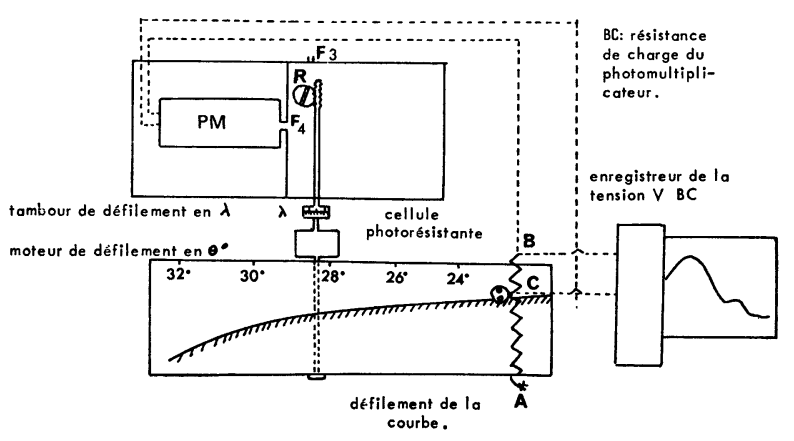

Fig. 7. - Montage de la correction de détection.

valeur maximum $B A$ pour la longueur d'onde $\lambda_{F 1}$ qui correspond au minimum de la fonction $T\left(\lambda_{F}\right)$ dans le domaine choisi. Pour toute autre longueur d'onde $\lambda_{F}$, on donne à $B C$ la valeur

$$
B C=B A \cdot \frac{T\left(\lambda_{F 1}\right)}{T\left(\lambda_{F}\right)} .
$$

III. Résultats expérimentaux. - 1 . CORRECTION D'EXCITATION. - Avec une deuxième thermopile située à la place de la cuve à fluorescence, nous avons enregistré l'intensité lumineuse d'excitation sans et avec le système d'asservissement, et ceci pour les deux lampes utilisées. Dans chaque cas nous avons introduit, pour le visible, un filtre passe-haut coupant l'ultraviolet afin d'éliminer le deuxième ordre du réseau ; les figures 8 et 9

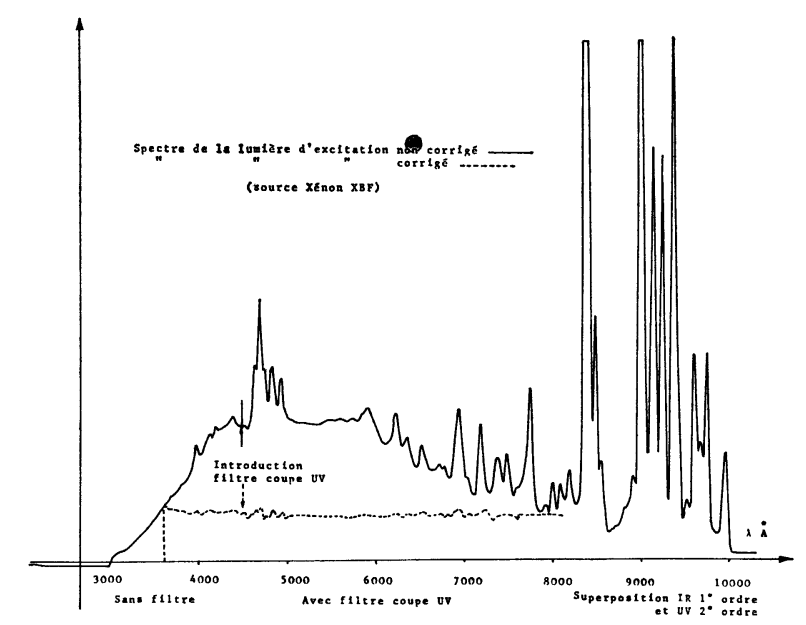

FIG. 8. - Spectres de la lumière d'excitation pour la lampe XBF.

représentent ces enregistrements. On peut constater que le flux corrigé subit encore des fluctuations de l'ordre de $40 \%$ avec l'arc au Xénon ; ceci est dû en particulier à la présence de raies introduisant de fortes pentes. Il 
faudrait pour améliorer le taux d'ondulation, soit diminuer la vitesse d'exploration, soit diminuer la constante de temps du système d'asservissement. Cette dernière est relativement importante (de l'ordre de $20 \mathrm{~s}$ ) due presque exclusivement au galvanomètre utilisé en régime amorti à cause de la faible résistance de la thermopile.

Avec la lampe quartziode, les fluctuations sont réduites à $17 \%$ pour l'ensemble du spectre (de 3000

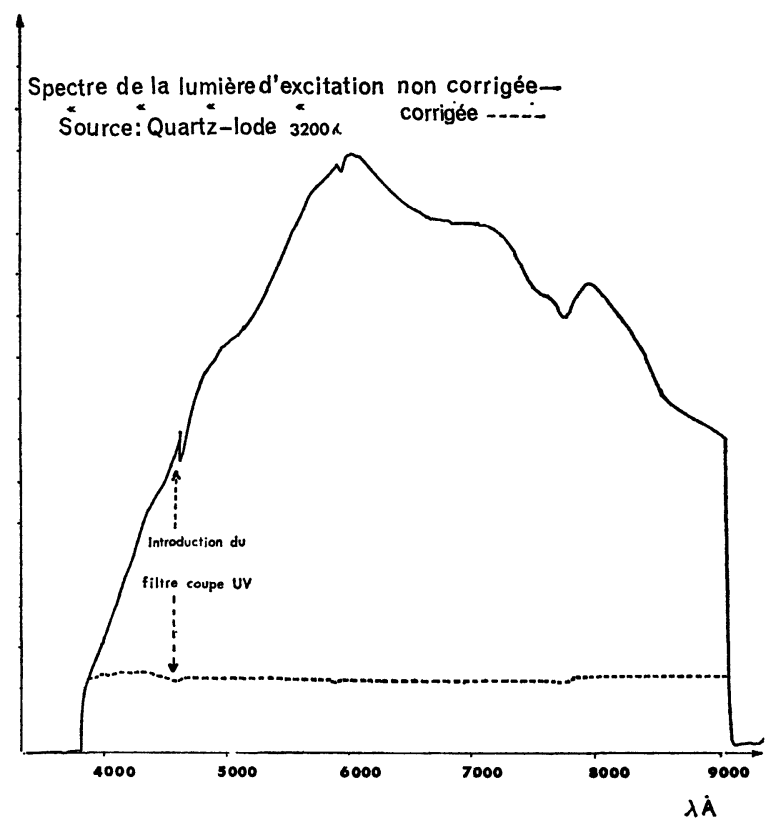

Fig. 9. - Spectres de la lumière d'excitation pour la lampe quartz-iode.

à $8000 \AA$ ) et de $12 \%$ de 4200 à $8000 \AA$. On peut constater d'autre part des zones (6000-7 $500 \AA$ par exemple) où les fluctuations sont réduites à quelques pour cent. Ce domaine est particulièrement intéressant puisqu'il correspond à une zone d'absorption de la chlorophylle.

Dans les deux cas, on peut constater qu'une grande partie de l'énergie lumineuse est perdue, la régulation étant effectuée autour de l'énergie minimum dans le domaine considéré. Ici encore la lampe quartziode est préférable à l'arc au Xénon pour le visible.

2. Correction en ANAlyse. - La figure 10 représente la sensibilité énergétique du système d'analyse et la courbe inverse qui est réalisée dans la correction. On peut constater que dans la partie $6000-8000 \AA$ la sensibilité varie très rapidement ; ceci est dû au fait que le réseau (blazé à $5000 \AA$ ) et le photomultiplicateur sont dans une région d'efficacité décroissante. Ceci pourrait être amélioré soit en prenant un réseau blazé à une longueur d'onde supérieure, soit en utilisant un photomultiplicateur ayant une réponse du type S 1 (7102 RCA par exemple). Cette dernière solution présente un inconvénient car ce photomultiplicateur, outre un bruit de fond et un courant d'obscurité importants qui néces- sitent un refroidissement, possède un rendement quantique très faible Imême vers le maximum rouge

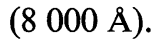

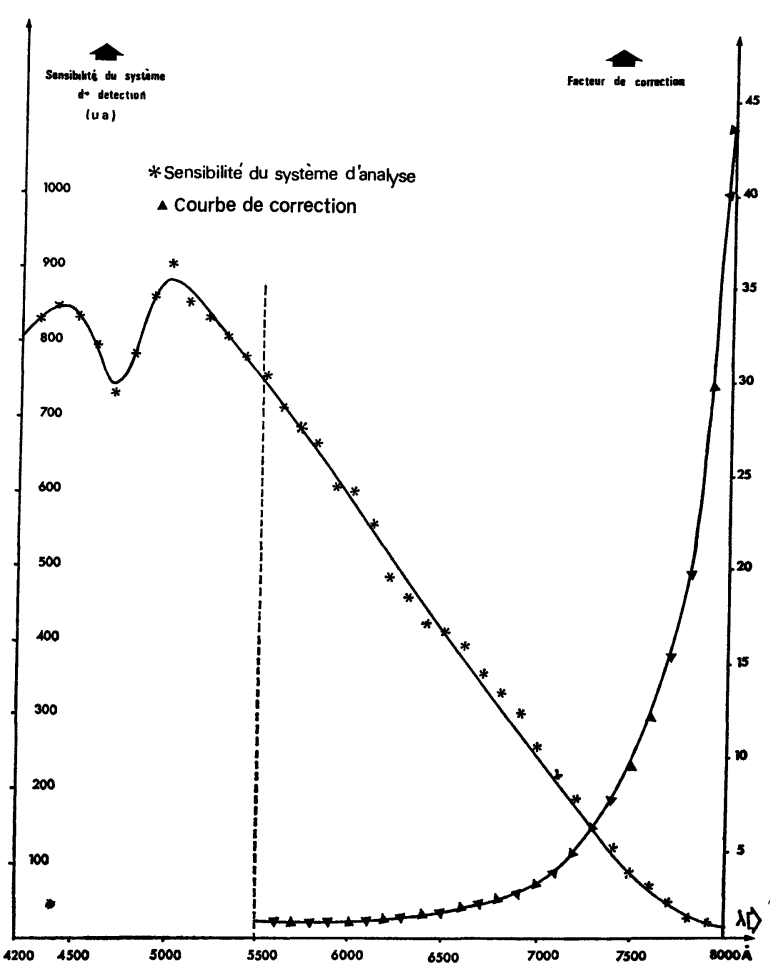

FIG. 10. - Courbes de sensibilité du système d'analyse et de correction.

3. Application a la CHLOROPHYLle. - Nous avons testé notre spectrofluorimètre avec une solution de chlorophylle a (benzène, concentration $5 \times 10^{-6}$ mole/ litre).

Spectre d'émission. - Les figures 11 et 12 représentent le spectre d'émission de la chlorophylle sans

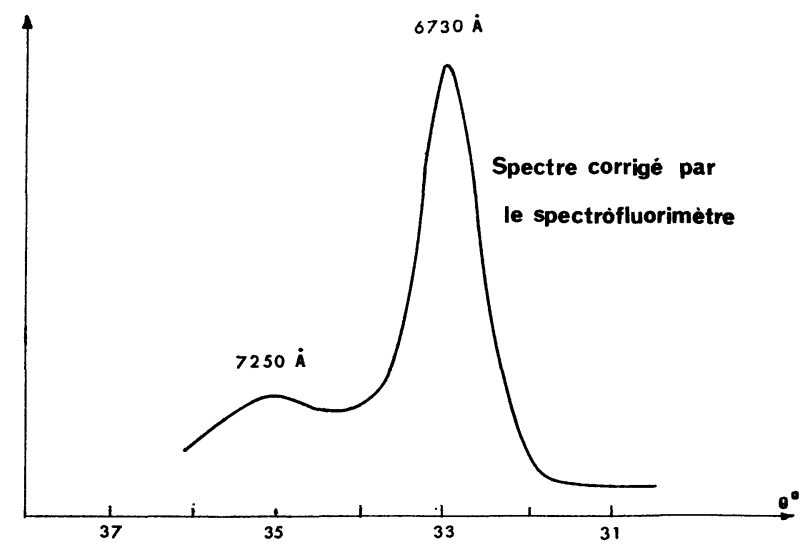

FIG. 11. - Spectre d'émission d'une solution de chlorophylle a corrigé par l'instrument.

correction, avec correction, par le calcul, et avec correction automatique. On constate que l'épaulement à 7250 , image de l'épaulement en absorption à 6160 (loi de Stokes) est bien présent dans le spectre corrigé [9]. 


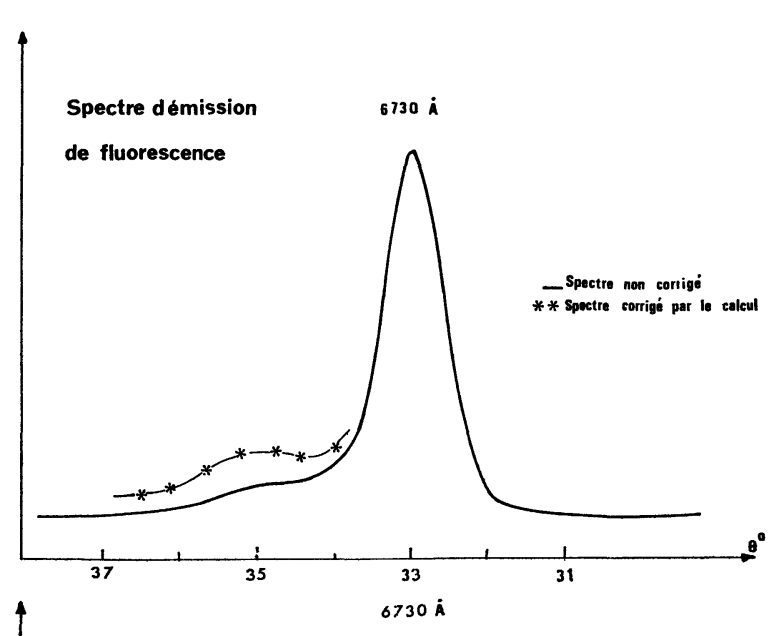

Fig. 12. - Spectres d'émission d'une solution de chlorophylle a sans correction et corrigé par le calcul.

Spectre d'action. - La figure 13 montre les spectres d'action avec et sans le système de stabilisation en excitation. On peut constater une différence très importante entre les deux enregistrements ; mais il est difficile de faire une comparaison simple avec le spectre d'absorp-

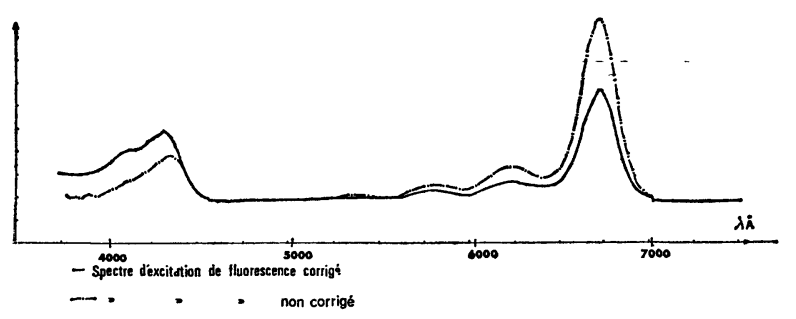

Fig. 13. - Spectres d'action pour une solution de chlorophylle a avec et sans correction.

tion à cause de l'effet de filtre interne d'une part, et d'autre part, du fait que le rendement de fluorescence est un rendement quantique, alors que la stabilisation est faite en énergie.

4. Sensibilité. - Bien que non adapté pour le travail dans le proche ultra-violet en excitation, nous avons fait un test de sensibilité avec le sulfate de quinine en solution sulfurique. Les conditions étaient les suivantes :

- tension du photomultiplicateur $1600 \mathrm{~V}$;

- alimentation de la lampe $110 \mathrm{~V}$;

- largeur des fentes en excitation : $2 \mathrm{~mm}$ (largeur de bande $50 \mathrm{~A}$ ) ;

- largeur des fentes en analyse : 1,5 $\mathrm{mm}$ (largeur de bande $30 \mathrm{~A}$ ) ; $10^{5} \Omega$

- résistance de charge du photomultiplicateur:

Le premier spectre (Fig. 14) correspond à une solution à $5 \mathrm{\gamma} / \mathrm{cm}^{3}$; la sensibilité de l'enregistreur est de $0,1 \mathrm{mV} / \mathrm{cm}$ ce qui correspond à un courant de $10^{-9} \mathrm{~A} / \mathrm{cm}$. Le signal de fluorescence a donc une amplitude maximum de $16 \times 10^{-9} \mathrm{~A}$ pour un courant d'obscu-

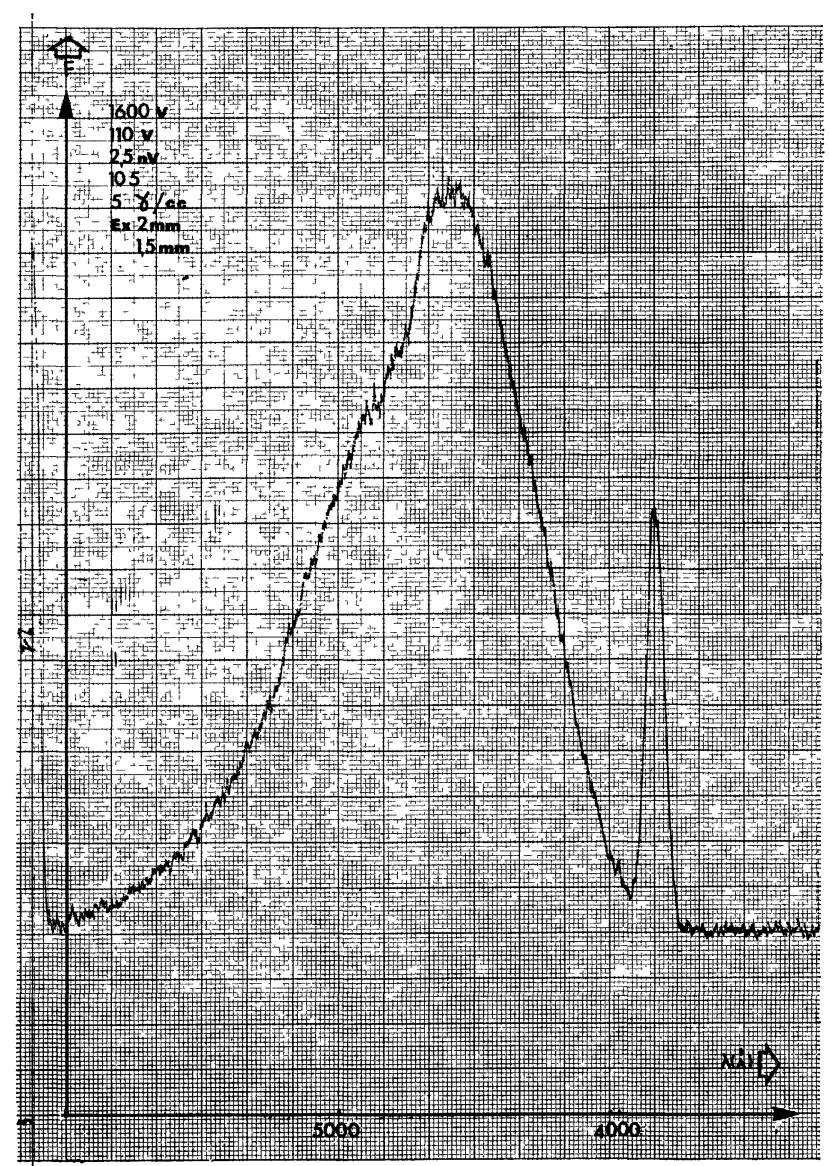

FIG. 14. - Spectre d'émission du sulfate de quinine à $5 \mathrm{\gamma} / \mathrm{cm}^{3}$.

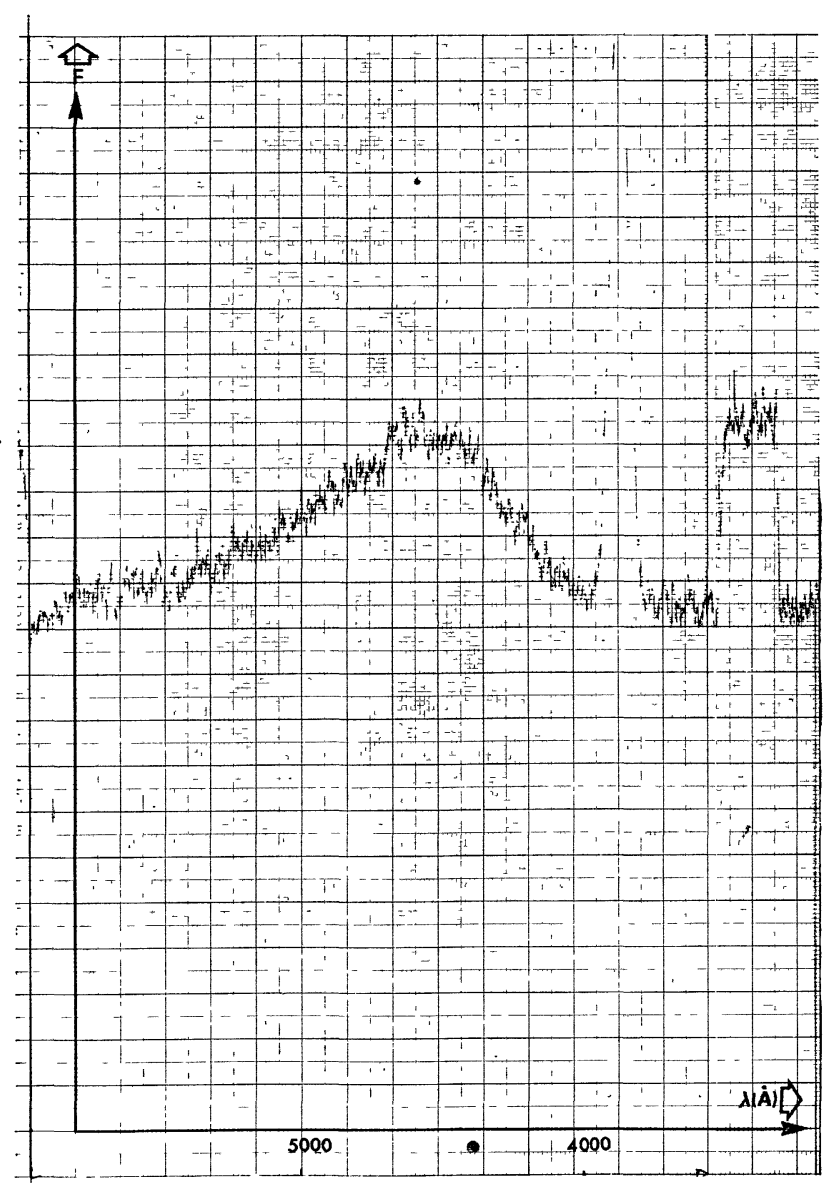

Fig. 15. - Spectre d'émission du sulfate de quinine à $0,5 \gamma / \mathrm{cm}^{3}$. 
rité de $5 \times 10^{-9} \mathrm{~A}$ et un bruit de fond de l'ordre de $5 \times 10^{-10} \mathrm{~A}$ crête à crête.

Le deuxième spectre (Fig. 15) correspond à une solution à $0,5 \gamma / \mathrm{cm}^{3}$, pour une sensibilité de $4 \times 10^{-2} \mathrm{mV} / \mathrm{cm}$, soit $4 \times 10^{-10} \mathrm{~A} / \mathrm{cm}$. Le signal de fluorescence a une amplitude maximum de $2 \times 10^{-9} \mathrm{~A}$ pour un courant d'obscurité de $5 \times 10^{-5} \mathrm{~A}$ et un bruit de fond de l'ordre de $5 \times 10^{-10} \mathrm{~A}$ crête à crête.

\section{Bibliographie}

[1] MC Carthy (W. J.), Winefordner (J. D.), In fluorescence (George G. Guilbault Ed.), DeKKeR (M.), Inc. New York, 1967, 371.

[2] Stupp (E. H.), Rev. Sci. Instrum., 1962, 33, 1215.

[3] Krauzman (M.), Kara (R.', J. Phys. Appl., 1966, 1, 247.

[4] Borresen (H. C.), Acta Chem. Scand., 1965, 19, 2089.

[5] Witholt (B.), Brand (L.), Rev. Sci. Instrum., 1968, 39 9, 1271 .
[6] Rosen (P.), Edelman (G. M.), Rev. Sci. Instrum., 1965, $366,809$.

[7] Haugen (G. R.), Marcus (R. J.), App. Opt., 1964, 3, 1049.

[8] Howerton (H. K.), In fluorescence (George G. Guilbault Ed.), DekKer (M.), Inc. New York, 1967, 233.

[9] Vernon (L. P.), Seely (G. R.), The chlorophylls Acad. Press, 1966. 